\title{
Negotiations in Spatial Reasoning Applications
}

\author{
Panel-chair: E. Jungert, FOA, Sweden \\ Panelists: S.-K. Chang, University of Pittsburgh, Pittsburgh, USA, R. Laurini, INSA and University of Lyon, Lyon, France, \\ and K. Ohlsson, Luleå Technical University, Luleå, Sweden
}

\section{Background}

The full potential of Internet and its use has not yet been completely realized. However, the evolution of the net has, by now, come to a point where some new applications, which will have a strong impact on how we will use computers in the future, have already been identified. Among these aspects the use of the web for improvised meetings is of particular interest. This is due to the fact that, for instance, in physical planning situations will occur where users, or rather groups of users, need to negotiate with respect to various urgent problems. In particular, this is especially complicated in such applications, that in one way or the other, are concerned with spatial information. That information may not just map geographical space but other types of space must be of concern as well, such as the internal part of a human body or a building. What makes the situation so complicated in spatial applications is that the information may come from multiple sources (e.g. sensors) that make it necessary to apply operations that quite often will be very complex. Examples of such complex operations are data fusion and spatial reasoning. Temporal aspects will complicate the situation even further.

A consequence of all the above is that individual users in a user group may not always agree to the interpretation or status of a particular state of the application and neither do they agree to the decisions that must be made as a consequence of the current problem. For this reason, it will be necessary to negotiate to get an outcome of the problem being discussed. This will require various tools for visualization and fusion of all available and required information in the application of concern. A further consequence is also that new types of visual languages must be designed to support negotiated spatial and/or visual reasoning.

\section{Groupware for spatial negotiations (R. Laurini)}

With some respects, this kind of web-based spatial negotiation system can be seen as an extension of a groupware, or a Computer Supported Collaborative Work (CSCW) system for participatory design in urban and environmental projects [1].

\section{Intelligent Information Retrieval (S. K. Chang)}

With the rapid expansion of the wired and wireless networks, a large number of soft real-time, hard real-time and non-real-time sources of information need to be quickly processed, checked for consistency, structured and distributed to the various agencies and people involved in information handling. In addition to databases, it is also anticipated that numerous web sites on the World Wide Web will become rich sources of information.

However, since too much information is available, information related to an important event could be missed because people are unable to track the manifestations of an unfolding event across multimedia sources over time. What is needed, from the information technology viewpoint, is an Active Multimedia Information System (AMIS) capable of processing and filtering multimedia information, checking for semantic consistency, discovering important events, and structuring the relevant information for distribution.

In practice, a significant event often manifests itself in different media over time. Therefore, if we can index such manifestations and dynamically link them to one another, then we can check for consistency and discover relevant information.

This dynamic indexing technique can be based upon the theory of active index [2]. Horizontal reasoning and vertical reasoning techniques can then be applied to consistency checking. For intelligent information retrieval from the World Wide Web, we proposed the WAG (Web-At-a-Glance) system [3] which can assist the user in creating a customized database by gleaning the relevant information from web sites containing 
multimedia data. WAG first interacts with the user to construct a customized conceptual vicw of the web pages pertinent to the user's interests, and then populates the database with information extracted from the web pages. We will discuss how a visual language can be developed to assist the user in an interactive approach of horizontal and vertical reasoning for intelligent information retrieval.

This panel will discuss these aspects and also try to forecast the future evolution of web oriented meeting systems. The panel will also focus on how negotiations can be performed with respect to the spatial and temporal applications, that will be of concern, from computer science, cognitive and sociological viewpoints.

\section{References:}

[1] R. Laurini, "Groupware for Urban Planning". COSTUCE C4 Workshop Information Systems and Processes for Urban Civil Engineering Applications. Rome, November 21 - 22, 1996, University of Rome "Tor Vergata"

[2] S. K. Chang, "Towards a Theory of Active Index", Journal of Visual Languages and Computing, Vol. 6, No. 1, March 1995, 101-118.

[3] T. Catarci, S. K. Chang, D. Nardi and G. Santucci, "A Prototype Web-At-a-Glance System for Intelligent Information Retrieval", Proc. of SEKE'97, Madrid, Spain, June 18-20, 1997, 440-449. 